\title{
COMPUTER-AIDED VISUAL COMMUNICATION FOR WAY-FINDING IN EMERGENCY IN INDOOR ENVIRONMENT
}

\author{
Po-Han Chen \\ Assistant Professor \\ School of Civil and Environmental Engineering, \\ Nanyang Technological University, \\ Singapore 639798 \\ cphchen@ntu.edu.sg
}

\author{
Nguyen Thi Lan Truc \\ Master of Engineering Student \\ School of Civil and Environmental Engineering, \\ Nanyang Technological University, \\ Singapore 639798 \\ nguy0050@ntu.edu.sg
}

\begin{abstract}
Effective and rapid finding a way out in emergency is always a problem for occupants who get trapped in indoor environment such as inside buildings or vessels. In such a case, rescuers often rely on emergency signs or verbal communication to direct trapped people to the exits. However, many problems could arise on the move such as mobile signal interruption, low visible scene caused by dense smoke, etc. These problems might prevent the rescuers to communicate with Command Centre as well as to occlude them to see the emergency signs and passageway clearly. Additionally, a complex spatial layout with occluded spaces will add more difficulties in decision the correct way to take.

To address these problems, this paper introduces an approach which uses visual communication with the aid of Virtual Reality (VR) and Augmented Reality (AR) technology to provide rescuers the effective and efficient direction messages during rescue operation. Content of the visual support is determined and generated corresponding to the rescuer's information (current position of rescuer, rescuer's head orientation) and current environment visibility. It is made up by combining a dynamic You-Are-Here (YAH) map superimposing on either real environment or virtual environment. Dynamic YAH map which presents the comprehensive spatial layout will be rotated in a way that the map's view is always congruent with the real layout with respect to the reader's viewing perspective. In case of low visible scene, a virtual environment (VR mode) is retrieved to enable the rescuer to identify the current places and the escape routes. Alternatively, if scene is clear the user could be able to see the real scene (AR mode) while still keep track of superimposing information (computergenerated). The generated visual representation will be then displayed on rescuer's Head Mounted Display (HMD) in realtime manner.

This kind of visual effect could reduce mental interpretation and the user's manipulation in reading supporting information of an emergency task. Therefore, it could speed up the time for decision making in emergency situations or during rescue operation as well.
\end{abstract}

\section{KEYWORDS}

Virtual Reality, Augmented Reality, Indoor Environment, Visualization 


\section{AIMS}

Effective and efficient rescue after a disaster is always a demanding, difficult task and a matter of life and death for both outdoor and indoor environments. In such a case, rescuers need to take the immediate and accurate responses based on disaster situations to direct trapped people to the safe and nearest exits. Technical supports are manifold, however this matter is still a practical problem needed to address, especially the problem of how to find a way out in a short time during emergency situation in indoor environments.

Due to the fact that rescue in indoor environments has to cope with various difficulties with limited resources available in search and rescue such as Global Positioning System (GPS), etc. Rescuers normally rely on verbal communication which uses mobile communication devices or walkie-talkie to communicate with and receive instructions from Command Centre [1]. This way of communication is very effective to provide direct and fast instructions. Rescuers could then follow the instruction and act promptly. Nevertheless, verbal communication could turn out to be infeasible in indoor environment where signal cannot reach. Alternatively, visual communication may take its turn to show occupants how to find ways out by seeing emergency signs like exit signs, arrows and You-Are-Here (YAH) maps. However, these signs could not be viewed clearly in smoky scene or in case of panic arising. Besides, some signs are not self-explanatory hence it takes time to interpret. YAH maps are one example. These maps are placed permanently on the wall with a fixed orientation in any locations [2]. Readers should rotate their heads or using mental interpolation to understand properly.

To address the problems of way-finding in emergency in indoor environments, a visualization system is proposed and developed based on the advantages of visual communication in way-finding in emergency and some modern technologies. The system combines Augmented Reality (AR), Virtual Reality (VR) and indoor trackers to provide effective and efficient assistance to rescuers. The primary aim of the system is to help the rescuers to navigate easily and find the way out in short time in emer- gency, regardless of the visibility of the indoor environment. By using such kind of system, the rescuers just follow the provided information without any elaborated manipulation or mental interpretation.

\section{SIGNIFICANCES}

This research will significantly provide an effective support for the rescuers who are immediately involve in the rescue or evacuation process. By using the visualization system, the rescuers could easily find the way out in a short time regardless the environment visibility of the disaster area. This research is not only appropriate for practical cases but also applicable for any training purposes.

The approach of using visual communication will provide a concise and self-explaining visualization with which the rescuers could easily to perceive the provided information in less time. Virtual Reality and Augmented Reality technologies play an important role to solve the problem of a change in environment visibility during rescue operation. If scene is covered by visual noise such as smoke then VR will provide a virtual scene which is the $3 \mathrm{D}$ model of the real one. Otherwise, AR mode will serve support information superimposing on the real scene.

The most significance in this research is the use of indoor tracking technologies which are particularly tailored for indoor environments where GPS cannot reach. With positioning tracking engine and orientation tracker, the current position of a rescuer and his current moving orientation are obtained in real-time. These data will be used to generate the real-time 2D and/or 3D visualization in accordance with actual position and moving orientation of the rescuer.

\section{METHODS}

Methods used to develop the visualization system are as follows:

- To determine the proper content and format of the information to be displayed to the rescuers.

- To develop and integrate the visualization system

\subsection{Determining the Proper Content and Format of the Information for Display}

In indoor environment, visualization support for way-finding is often found as graphical signs in- 
forming occupants of the way to certain places. These signs are (1) symbols naming objects (2) YouAre-Here maps locating object relating to each other (3) a network of graphical arrows indicating the direction to the nearest exit [3].

Graphical symbols, maps and networks are designed to meet three distinct functions to assist way-finding [3]:

- To identify places and routes

- To map the spatial layout of an area

- To direct occupants and passengers to route

Therefore, these signs are employed in this research to develop the graphic features for way-finding directions.

In this research, all information to assist way-finding should be appeared on one screen hence they should be placed in an appropriate order. A good visualization will enhance the readers to quickly perceive the provided information. Literature on visual graphic communication provides a rich knowledge-based for effective design criteria[2,4]. Among them, some criteria are for determination the task-specific visualization for emergency situation. They are completeness, perceptibility, semantic clarity and convenience.

Completeness: All information that is necessary to fulfill the way-finding task is represented on the visualization. FigureError! No text of specified style in document.-3 is an example of the content for smoky scene which is the combination of a YAH map and a 3D virtual scene. The YAH map shows the spatial layout of the disaster area. On the map, the current position symbol and the routes are marked prominently to enable the rescuer to identify where he is and which escape way to the nearest exit he should take. Since people often make mistake in identifying the place as well as the passageways with 2D layout [5], therefore 3D scene is provided in addition to adapt the user viewing perspective. 3D scene will be appeared in two alternatives: real scene and virtual scene in order to solve the environment visibility problem. Real scene will be used for clear environment and virtual scene will be used for visual noise environment.
Perceptibility/syntactic clarity/visual clutter: As shown in FigureError! No text of specified style in document.-3, all relevant graphics of the information represented in the visualization are appear in readable scale at appropriate places hence it is easily perceptible. Visual clutter which is unnecessary graphic features should be reduced in minimum. For instance, furniture symbols and technical symbols appearing on 2D map should be eliminated in order to make the map more readable (Figure-1). This criterion is also applied for modeling the $3 \mathrm{D}$ virtual scene as shown in Figure.

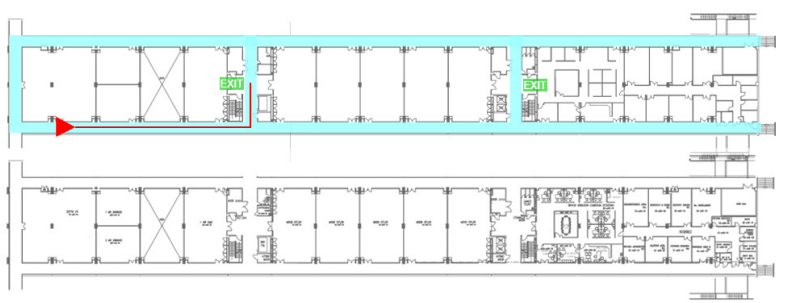

Figure-1. Unnecessary objects should be eliminated in the YAH map

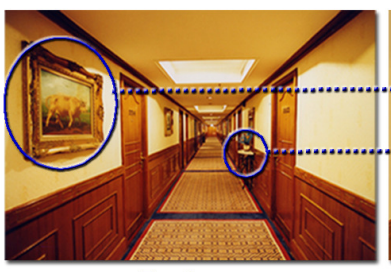

Real scene
3D model

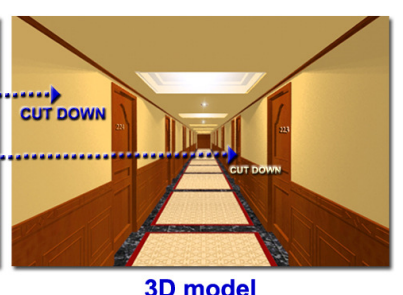

Figure-2. Cutting down visual clutter in the 3D virtual scene

Semantic clarity: As shown in FigureError! No text of specified style in document.-3, contexture use of the symbols and map features are simple but imbued with meaning. In other words, they are selfexplanatory. For instance, by seeing the red arrow on YAH map the rescuer could recognize where they are and their viewing direction at present.

Convenience: All information are displayed and updated in real-time during rescue operation to facilitate rescuers to achieve their task at any time. Real-time YAH map alignment is a crucial issue to 
enhance way-finding with ease. The map will be rotated each time the user changes his orientation in a way that the map is always aligned with the real layout. In other words, the map layout is always congruence with the user perspective. This work will reduce significantly necessity for mental and head rotation. Therefore, the users could recognize the way out easily. An example of real-time YAH map alignment could be seen in FigureError! No text of specified style in document.-2.
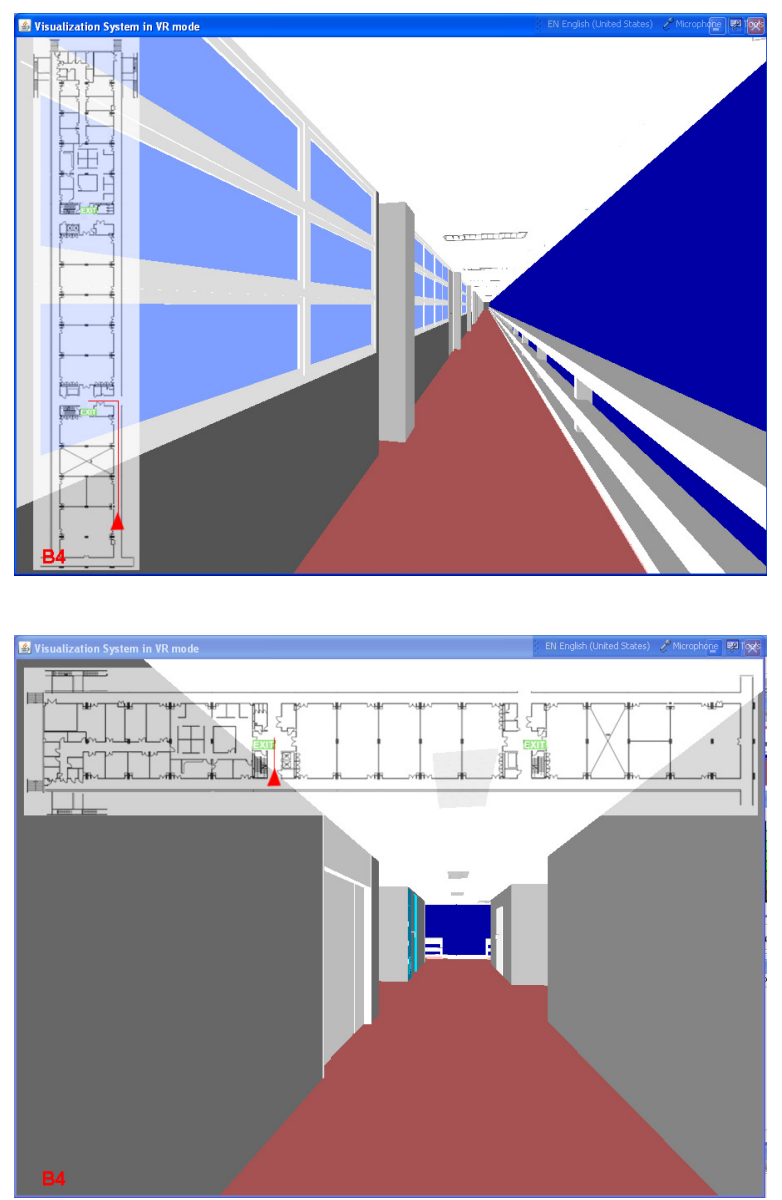

FigureError! No text of specified style in document.-2. YAH map will be rotated each time the user changes his orientation in a way that the map is always aligned with the real the layout

Ultimately, content of the information to be displayed to the rescuers is the combination of a YAH map superimposing on a 3D scene. On the map, current position of the rescuer, his viewing orientation and possible escape routes are marked prominently. Name of the floor and exit locations are also explicitly indicated. The 3D scene will be switched from real scene to virtual scene depending on the environment visibility. During navigating in the indoor environment, the YAH map will be rotated in accordance with the user viewing perspective.

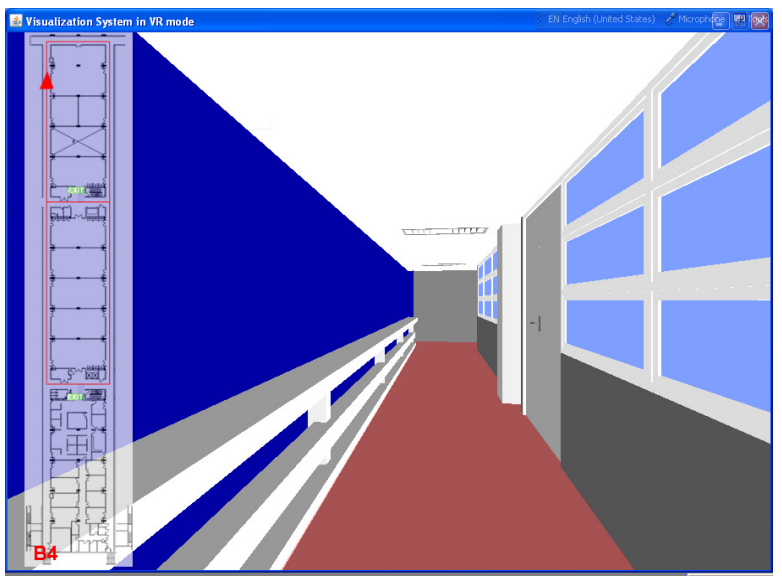

FigureError! No text of specified style in document.-3. Content of the information to be displayed to the rescuers in case of smoky scene

This research employs Virtual Reality technique and Augmented Reality technique in representing the visualization for virtual scene and real scene, respectively. Therefore the content will appear in either Virtual Reality display or Augmented Reality display upon the environment visibility. Virtual Reality display is the combination of the YAH map superimposing on the virtual scene. Augmented Reality display is the combination of the YAH map superimposing on the real scene which is recorded by a video mounted on the user's head.

\subsection{Visualization System Development}

\subsubsection{General Framework Development}

The general framework as shown in Figure-4 represents comprehensive picture of the visualization system including system's features and activities. Input data of the system are dynamic data and pre- 
defined data. Dynamic data are current position of a rescuer and viewing orientations which are real-time retrieved from indoor trackers. Pre-defined data are floor layouts and 3D model of the environment. After input step, possible escape routes and YAH map are generated in turn. Based on the current environment visibility, corresponding scene is selected. Virtual scene will be used for Virtual Reality display and real scene is for Augmented Reality display. All graphic features are then integrated and rendered for output display.

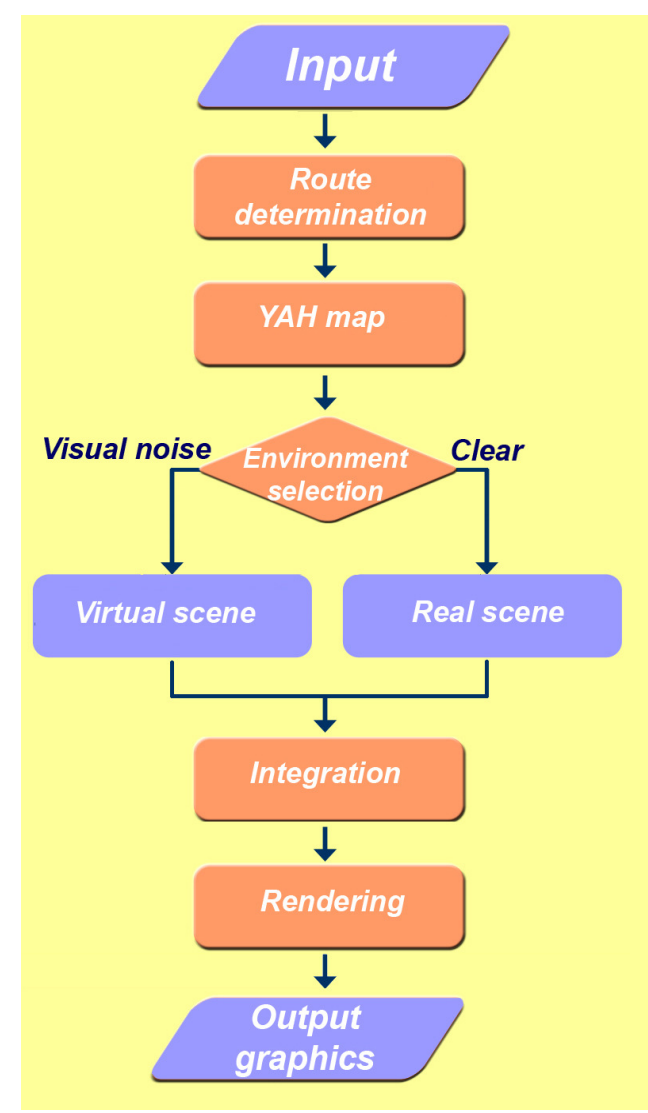

Figure-4. General framework of the visualization system

Since this framework is generic hence it could be applied for way-finding in emergency for other in- door environments by changing the pre-defined input data.

\subsubsection{Visualization System Integration}

Based on the framework, a specific visualization system could be developed with specific data by integrating the system components including corresponding hardware and software. Hardware of the whole system will have (1) a compact laptop or a PDA, (2) an orientation tracker, (3) a video seethrough Head Mounted Display (HMD). In this research, Ekahau positioning engine is used to track the current position of the rescuer in real-time via wireless signal. This engine is installed into the laptop or the PDA which has Wi-Fi feature. All hardware are hooked up to perform the visualization task.

Java and Java3D programming language is used to develop the system's functions as well as to work out with tracking devices. Output of the system will be displayed on an Applet window.

\section{RESULTS}

A practical application of the visualization system has been developed and demonstrated at block N1 in Nanyang Technological Campus. 2D layouts and 3D model of the building is prepared in advance and used as input data. The system is performed along the passageway of the level B4.

The following snapshots in Figure-5(a) (b) (c) (d) are the Virtual Reality displays generated by the visualization system for visual noise scene. Position data is used to determine the position of the rescuer in 3D virtual scene and on 2D layout as well as to generate the possible escape routes. As shown in four figures, the YAH map is rotated if the user changes his viewing orientation.

FigureError! No text of specified style in document.-6 (a) (b) contains two snapshots for Augmented Reality displays. In this mode, the user is able to see the real scene while following the provided information on the YAH map. 


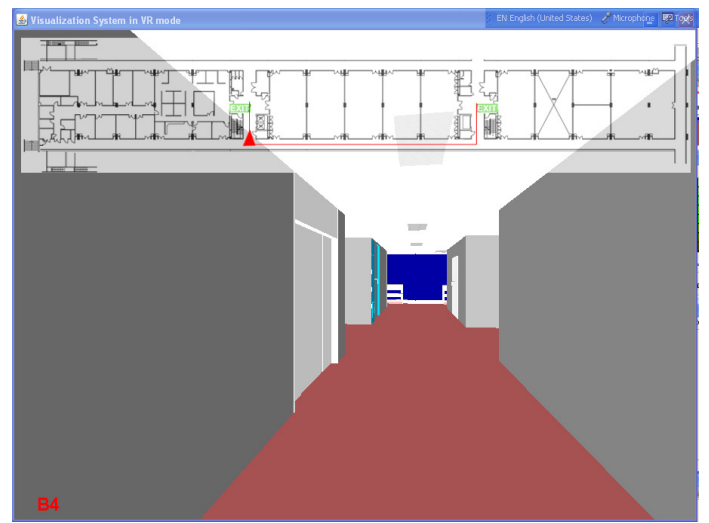

(a)

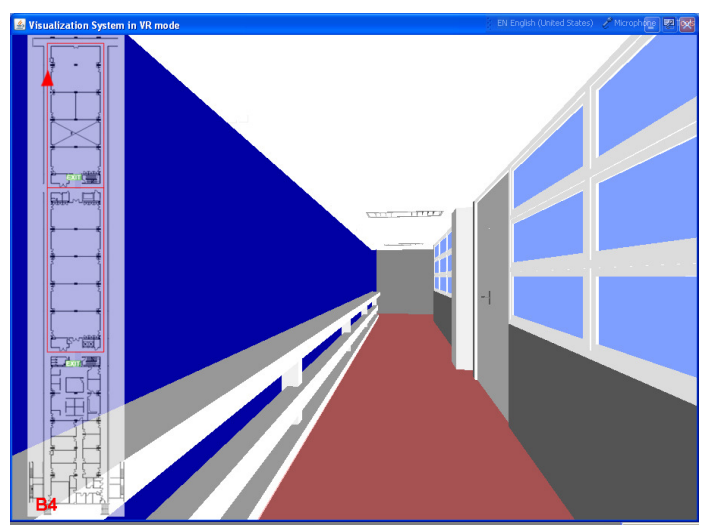

(c)

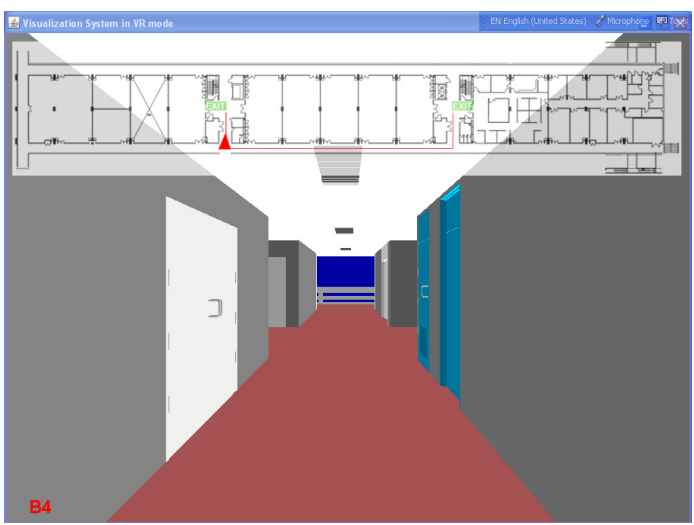

(b)

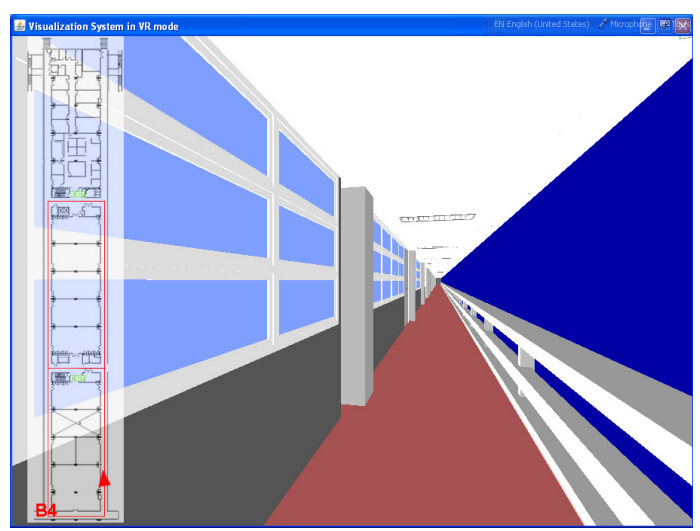

(d)

Figure-5. The visualization for Virtual Reality display

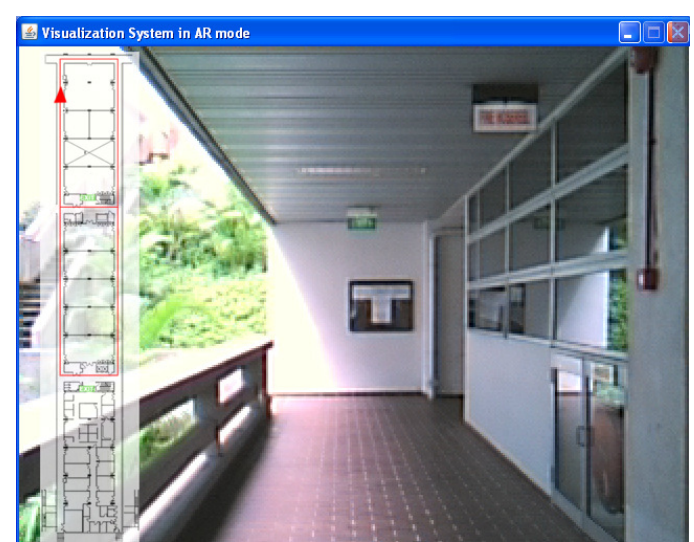

(a)

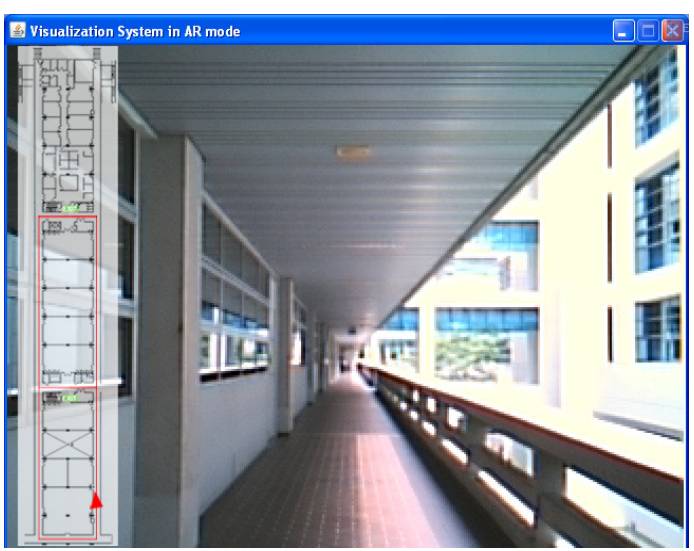

(b)

FigureError! No text of specified style in document.-6. The visualization for Augmented Reality display 


\section{CONCLUSIONS}

This research contributes an effective and practical method to support rescuers to achieve the rescue task in the context of way-finding in indoor environments. Through computer-aided visual communication approach, this research is among the first of its kind.

Since the visualization system will automatically generate and provide real-time visualization support to the rescuer through HMD. Hence the rescuer could feel free to use the system with ease. Moreover, the visualization content is investigated based on human-centric factor, which concentrates on easy-to-perceive aspect. Therefore, the rescuers could quickly perceive and grab the provided information on HMD.

Due to the constraint in time and technology, this research has just completed the demonstration work in a simple environment with the use of some nonspecialized devices. However, this research has provided a base for the future work or other researches with regard to using computer-aided visualization in rescue.

\section{REFERENCES:}

[1] Lemmel, M.C. Interactive information and communication system for emergency applications on the example of fire brigades

[2] Klippel, A.; Freksa Ch.; Winter, S. You-Are-Here Maps in Emergencies - The danger of getting lost, in Journal of Spatial Science, 51 (1)

[3] May, M.: Wayfinding, Ships, and Augmented Reality in: Peter Bøgh Andersen and Lars Qvortrup (eds.) Virtual Applications: Applications with Virtual Inhabited 3D Worlds. (Springer Verlag, to appear 2003).

[4] Jankovic, M; IN \& Palij, M 1982, Principles of spatial problem solving, Journal of Experimental Psychology: General, vol. 111, no. 2, pp. 157-75

[5] Kleiner and Goebelbecker, M., Rescue3D: Making rescue simulation attractive to the public, Technical report Institute for Computer Science, University of Freiburg, 2004. 